\title{
ISCHAEMIC NECROSIS OF THE ANTERIOR TIBIAL MUSGLES DUE TO FATIGUE
}

\author{
J. Rowland Hughes, Liverpool, England \\ From the Liverpool Open-Air Hospital for Children, Leasowe \\ Formerly of the Orthopaedic Service, the Royal Air Force
}

Ischaemic necrosis of the lower limb muscles is well recognised as a complication of injury and recorded cases show that, as in the upper extremity, isolated muscles or muscle groups may be affected (Bruce 1940, Parkes 1945, Wood Power 1945). Traumatic ischaemic necrosis of the anterior tibial group of muscles may thus occur; but it is less widely appreciated that such necrosis may also arise when there has been no injury at all.

Hill and Brooks (1936) described muscle contractures arising spontaneously in patients suffering from haemophilia, but it is uncertain whether any of these were due to ischaemic muscle necrosis. Horn (1945) recorded two cases of contracture of the tibialis anterior and the long extensors of the toes in young healthy soldiers after strenuous exercise, and he quoted Vogt as having recognised the syndrome in soldiers after marching. This relationship between exercise and circulatory disturbances of the anterior tibial muscles was stressed by Sirbu, Murphy, and White (1944), who described the case of a young soldier in whom ischaemia of the anterior tibial group of muscles was believed to have been attributable directly to route-marching. Mr J. C. Scott of Oxford tells me that he has knowledge of four such cases, occurring in healthy young adults after strenuous and unaccustomed activity. There can be little doubt, however, that many cases have passed unrecognised, particularly in the acute phase, and this is borne out by the cases now reported.

\section{GASE REPORTS}

Case 1. Sergeant C. History-On September 13,1945, after playing football, he felt that his " right knee was somewhat stiff." During the game he had experienced no cramp or pain in the leg and suffered no injury. He had not played football for several months before this game, but his health had been perfect and there had been no previous symptoms. The next day he complained of pain in the whole of the right lower leg and he was reluctant to move the ankle joint. A surgeon diagnosed acute tenosynovitis of the tibialis anterior and applied a viscopaste bandage. The next day the patient reported sick; the pain was unabated, and he was admitted to hospital.

Clinical examination-The patient was a healthy young adult, twenty-three years of age, and he showed these clinical features: 1) swelling of moderate degree over the antero-lateral aspect of the right lower leg as far as the ankle; 2) tense glossy redness of the skin over the middle third of the anterior tibial compartment; 3 ) extreme tenderness and induration of the whole of the anterior tibial compartment, with a little tenderness over the tibialis anterior at the ankle joint; 4) absolute reluctance to perform any active movement of the ankle joint, due presumably to pain. There was no pallor or cyanosis of the toes, no sensory loss, and no evidence of cardio-vascular or other systemic disease. The temperature was $99.2^{\circ} \mathrm{F}$; the blood sedimentation rate was $28 \mathrm{~mm}$. in the first hour (Westergren); the total white cell count was 11,600 per cu. $\mathrm{mm}$.

A diagnosis of cellulitis was made and the limb was immobilised in a Thomas' bed knee splint. The pain and discomfort settled gradually and by September 29 the tenderness, swelling, and redness had disappeared, but there was still slight evening pyrexia; the blood sedimentation rate was $14 \mathrm{~mm}$. in the first hour. At no time did radiographic examination show evidence of bone injury or disease. Examination on October 4 showed that the muscle belly of the tibialis anterior was abnormally hard, from a level two inches below the tibial tubercle to the junction of the middle and lower two-thirds of the leg. The other muscles of the anterior tibial group were not quite so hard. Active extension of the ankle joint was limited just below the right angle and it was weak. Plantar-flexion was restricted by contracture of the tibialis anterior and to a lesser extent by contracture of the long extensors of the toes. The peroneal muscles were acting normally. There were no vascular or sensory changes in the foot.

Biopsy-October 11, twenty-eight days after the onset of symptoms. The deep fascia of the anterior tibial compartment, and the muscle sheaths, were fused into one thick sheet. Incision revealed that the muscle was pale pink-grey in colour, hard, and without contractile response to mechanical stimulation. 
There was little response to direct galvanic stimulation, and no response to strong faradism. It was noted that there was venous oozing from the affected group of muscles. Part of the tibialis anterior muscle was taken for section, and also, for purposes of comparison, a small piece of the gastrocnemius muscle which showed a healthy brick-red colour and was briskly contractile. Histological examination of the affected muscle showed gross changes typical of ischaemic necrosis (Fig. 1).

Progress-The patient was fitted with a toe-elevating spring which he wore for five weeks. Follow-up examination in March 1946 showed persistent hardness of muscles in the middle third of the anterior tibial compartment. The total range of active movement of the ankle was 15 degrees. Contracture was maximal in the tibialis anterior and was present to a much less degree in the extensor hallucis longus and the extensor digitorum longus. There were no sensory changes. The patient walked well. His only complaint was of occasional aching in the limb.

Case 2. Leading-Aircraftman S.-A young adult, twenty years of age, had complained of vague pain over the antero-lateral aspect of both lower limbs for nine months. After playing football on May 22, 1946, he noticed dull aching in front of his right lower leg; the whole leg and ankle joint felt stiff. At no time during the game did he experience pain or cramp; nor did he sustain injury. It was not until several hours after the game that symptoms arose. The next day the leg was swollen and very painful and he was admitted to hospital where he received a course of penicillin injections and sulphapyridine, followed later by faradism, radiant heat, and massage. No further details of the clinical condition are available but the treatment which was given suggests that the clinical picture resembled that of an acute inflammatory lesion. After two months he was transferred to ancther hospital.

Clinical examination-There was: 1) one inch wasting of the right calf; 2) a palpable woody hard area corresponding to the lower two-thirds of the tibialis anterior; 3) limitation of active and passive plantarflexion at the ankle joint due to contracture of the antericr tibial group of muscles; 4) marked weakness of active dorsiflexion of the ankle joint; 5 ) no more than a suspicion of active contraction of the tibialis anterior muscle, and a flicker of contraction in the extensor hallucis longus, but good contraction of the extensor digitorum longus and vigorous action in the peronei; 6) diminished sensation over the dorsum of the foot and front of ankle. There was no evidence of vascular impairment in the foot, no swelling, no erythema, no tenderness over the anterior tibial compartment, and no evidence of cardio-vascular or other systemic disease. Radiographic examination of the limb showed no abnormality. The blood sedimentation rate was $2 \mathrm{~mm}$. in the first hour (Westergren). The Wassermann reaction was negative. Electrical reactions showed negligible response to faradism and a weak response to galvanism in all anterior tibial muscles.

Biopsy-September 19, four months after the onset of symptoms, showed that the muscle fibres were yellow.white in colour and very resistant to cutting with a knife. The muscle had undergone mass necrosis and was surrounded by dense cellular fibrous tissue. The summary of the histological report was in the words: " the picture is that of ischaemic necrosis." (Fig. 2.)

Case 3. Leading-Aircraftman H., aged nineteen years, was admitted to the medical wards of a regional hospital on December 15, 1915, with swelling of the neck and face, dysphagia, and weakness of the legs. He gave a history of similar previous attacks, which on the last occasion had been diagnosed as acute thyroiditis. There was no history of injury or of cramp in the legs. While under investigation he developed crops of subcutaneous nodules, blue in colour, varying from inch to $1 \frac{1}{1}$ inches in diameter, situated over the chin, thigh, scrotum, forearms, and lower limbs. Each crop of nodules was associated with pyrexia, but blood cultures were negative. Investigations showed: blood count-red blood corpuscles, 4.5 million per cu. mm.; haemoglobis, 70 per cent.; white blood count, 9200 per cu. mm.; differential count, no significant change; blood sedimentation rate, $36 \mathrm{~mm}$. in the first hour (Westergren); Wassermann reaction negative; prothrombin time, normal; other investigations, negative. Biopsy of subcutaneous nodule-The summary of Professor G. R. Cameron's report on histological examination of one of the nodules is: "Some of the lesions are very similar to the acute stage of periarteritis nodosa but there is not much doubt that a rheumatic infection is the most likely cause of the condition." On February 28 , 1946, the patient complained of pain in both feet. The ankles and feet were swollen and there was a large nodule on the right sole. A further crop of nodules appeared, mainly in the limbs, associated with pyrexia of $101^{\circ} \mathrm{F}$. After a course of salicylates, the sysmptoms subsided. When he was transferred to an orthopiaedic centre he had been getting up for most of the day but he complained of weakness of the lower limbs.

Clinical examination-1) Active and passive plantar-flexion at both ankle joints were limited to 15 degrees by bilateral contractures of the tibialis anterior and extensor hallucis longus; 2) active dorsiflexion was very weak on both sides and was accomplisled mainly by the extensor digitorum longus; 3 ) there was woody hardness over the anterior tibial compartments in both legs; 4) the anterior tibial pulse was not palpable at the ankle joint; 5) there was no sensory disturbance in either leg. 
Biopsy of muscles-May 30, 1946. Both anterior tibial groups of muscles were explored. The fascia was thickened and fused with the sheaths of the underyling muscles, the latter being pale, hard, and fibrous, and not reacting to stimulation with the knife. There was faint response to direct faradic and galvanic stimulation. On both sides the tibialis anterior, and to a lesser extent the extensor hallucis longus, were affected; the entensor digitorum communis was involved mildly. Attempted exposure of the anterior tibial artery was made difficult by extensive fibrosis within the compartment. Specimens of the affected muscle and of the normal gastrocnemii were taken for section. Histological examination showed changes in the anterior tibial muscles similar to those of Volkmann's ischaemic contracture (Fig. 3). Progress-Plaster back slabs were applied at night and toe-elevating springs were used for walking. When last seen the patient was able to get about fairly well.

Comment-The exact nature of the systemic disease in this case is a matter of conjecture. Infarction of the anterior tibial muscles was not recognised so that the precise time of onset, in relation to the general disturbance, remains uncertain. It is possible that this occurred on February 28, 1946, when it was recorded that an attack of pyrexia was associated with pain and swelling of both ankles. However, further questioning, after transfer to an orthopaedic centre, revealed that from the time of onset of the illness the patient had experienced pain in both legs, particularly on the antero-lateral aspect where there was some swelling and redness. That these muscles should be picked out in this way, while the patient was confined to bed, illustrates their peculiar vulnerability to ischaemia even in the absence of physical exercise.

\section{CLINICAL FEATURES}

In the acute phase of the condition the clinical picture is sufficiently characteristic to permit accurate diagnosis. It is only failure to bear in mind the possibility of this lesion which accounts for the fact that so many cases pass unrecognised. During the first three or four hours the clinical features resemble those of tenosynovitis of the tibialis anterior, but there is no typical crepitation.

From twelve to twenty-four hours after the onset there is evidence of intense local reactionary inflammation (Lewis 1936). The skin over the anterior tibial compartment, particularly in its lower two-thirds, is tense, glossy, erythematous, and indurated. Swelling may extend as far as the ankle joint. There is often low pyrexia. The blood sedimentation rate shows a marked rise and there may be leucocytosis. It is not surprising, therefore, that most recorded cases were first diagnosed as cellulitis of the lcg. Horn (1945) described the onset of pain as sudden and severe, and occurring during exercise. This was not the typical history in the cases here recorded. These patients described a sensation of stiffness over the outer aspect of the shin, and later of aching, gradually mounting to severe pain which reached its greatest intensity from twelve to twenty-four hours later.

Peroneal palsy has been described, but it was not a corispicuous feature in these cases. In Case 1 there was complete loss of voluntary movement at the ankle joint during the acutely painful phase but there was no sensory change. In Case 2 there was a small area of residual hypoalgesia over the dorsum of the foot. The peroneal muscles were not involved. In this respect it is interesting to note that Case 3 was referred to the orthopaedic department because it was thought he had bilateral "drop foot "; in the sense that there was marked weakness of dorsiflexion this was true, but there was no evidence of a peroneal nerve lesion.

In established cases, when inflammatory reaction has been sucreeded by replacement fibrosis, the predominant symptom may be " weakness in the legs." There is obvious weakness of active dorsiflexion of the ankle joint, and often of the great toe. Active and passive plantar-flexion at the joint is limited by contracture of the tibialis anterior, and to a lesser degree by contracture of the long extensors of the toes. Palpation of the limb shows typical rigid firmness over the anterior fascial compartment, particularly in its middle third. The anterior tibial pulse may be diminished or absent-a sign which is of doubtful value.

Vol. $30 \mathrm{~B}$, No. 4 , NOVEMBER 1948 


\section{DISCUSSION}

Morbid histology-Obstructive vascular lesions in muscle may be of two main typesvenous and arterial. In each type the histological picture is characteristic (Brooks 1922, Middleton 1930, Griffiths 1940, Bowden and Guttman 1945). In all cases now under review

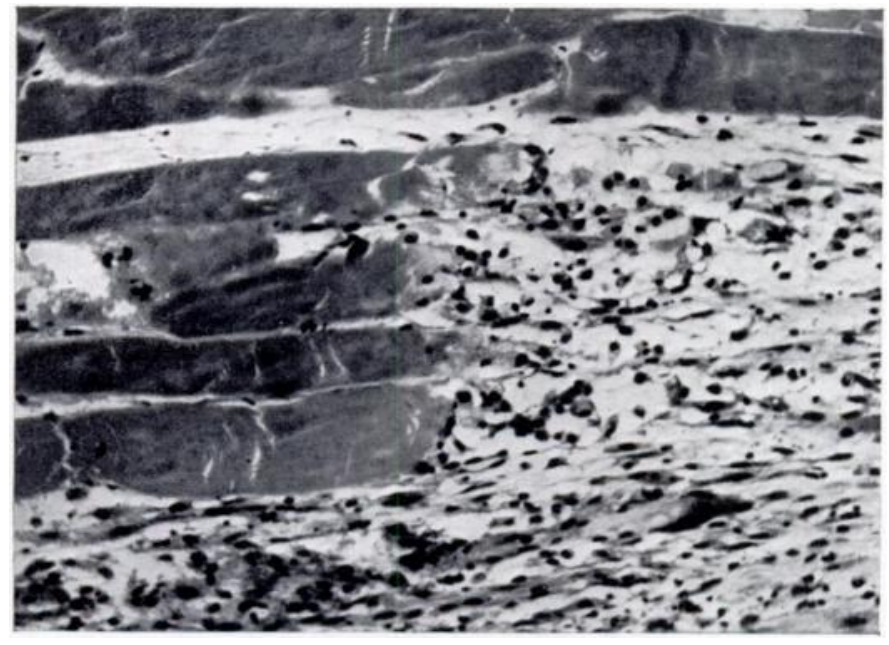

FIG. 1

(ase 1. Microphotograph showing peripheral zone of necrotic anterior tibial muscle $(\times 140)$. the microscopic picture showed that they were of the arterial type, similar to that which has been shown by Griffiths (1940) to occur in Volkmann's ischaemic contracture. Islands of necrotic muscle fibres are surrounded by a sea of fibrous tissue. The earlier stages show that the muscle fibres are swollen, enlarged, pale-staining, and with but few sarcolemmal nuclei (Fig. 1). Subsequently they become fragmented and shrunken, and they undergo active phagocytosis by foreign body giant cells (Fig. 4). The appearances after several months are exemplified in

Case 2 (Fig. 2): areas of muscle have undergone mass necrosis; while still retaining their structural outlines the fibres exhibit no nuclear staining; closely investing the necrotic muscle is a zone of dense fibrous tissue in which remnants of degenerated muscle fibres persist.

Professor Le Gros Clark (1946) produced identical lesions in the lower two-thirds of the tibialis anterior muscle of rabbits by ligating the main ressels, and demonstrated the remarkable degree of regeneration of muscle fibres which might occur within three weeks, provided that a stump of healthy muscle fibre remained. He showed convincingly that fine strands of protoplasm streamed down the muscle tubes into the necrotic area until, by the end of four months, the whole of the necrotic area was completely replaced by newly regenerated muscle fibres.

Regeneration of necrotic muscle in the human being.

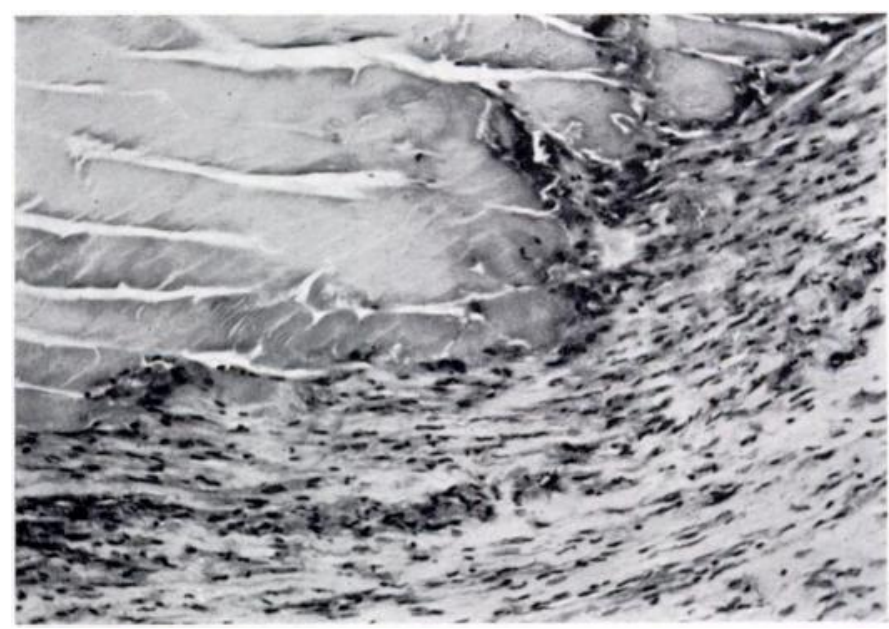

FIG. 2

Case 2. Muscle sequestrum surrounded by mature fibrous tissue; appearance four months after onset $(\times 100)$. Figs. 5 and 7 show unmistakably that such regeneration can occur also in human muscle. When asked for his opinion on these sections, Le Gros Clark pointed out that the difficulty in regeneration of human muscle, as compared with the muscle of rabbits, arose from its bulk; after ischaemic necrosis 
extensive fibrosis occurred before the regenerating fibres had time to cover the ground. Sprouting fibres soon met with the obstruction of fibrous tissue and became dammed up into large blobs of multinucleated protoplasm. These are shown typically in Fig. 5.

The possibility of primary arterial disease causing ischaemic necrosis of musclesIn view of the suggestion confidently put forward by Horn (1945) that chronic arterial disease preceded muscle necrosis, careful study was made of these sections by which to substantiate or refute this view. Two changes may be seen in the vessels included in the sections: first, there is thickening of the media, associated with a narrow lumen; second, there is diffuse perivascular cellular infiltration. One large vessel in Case 1 showed generalised intimal thickening. The clinical features in Case 3 suggested that arterial disease such as periarteritis nodosa

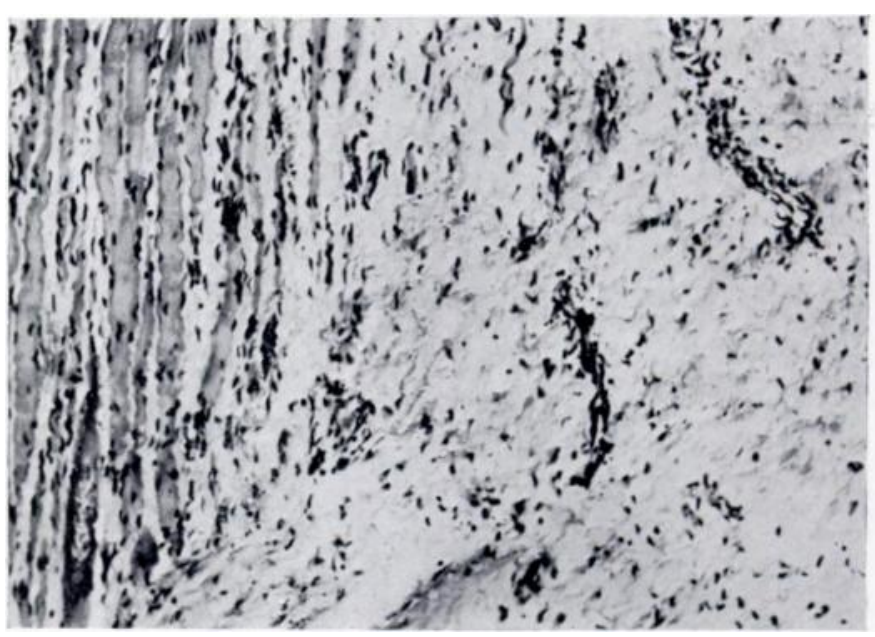

FIG. 3

(ase 3. Replacement of muscle by dense fibrous tissue $(\times 100)$. The changes are exactly similar to those in Volkmann's ischaemic contracture. might have been responsible for the infarction, but there was no histological confirmation.

The vascular abnormalities we have found can be explained fully as secondary changes. Death of functional muscle, and its replacement by avascular fibrous tissue, greatly reduces the vascular bed to be supplied. Small vessels naturally undergo involutionary thickening

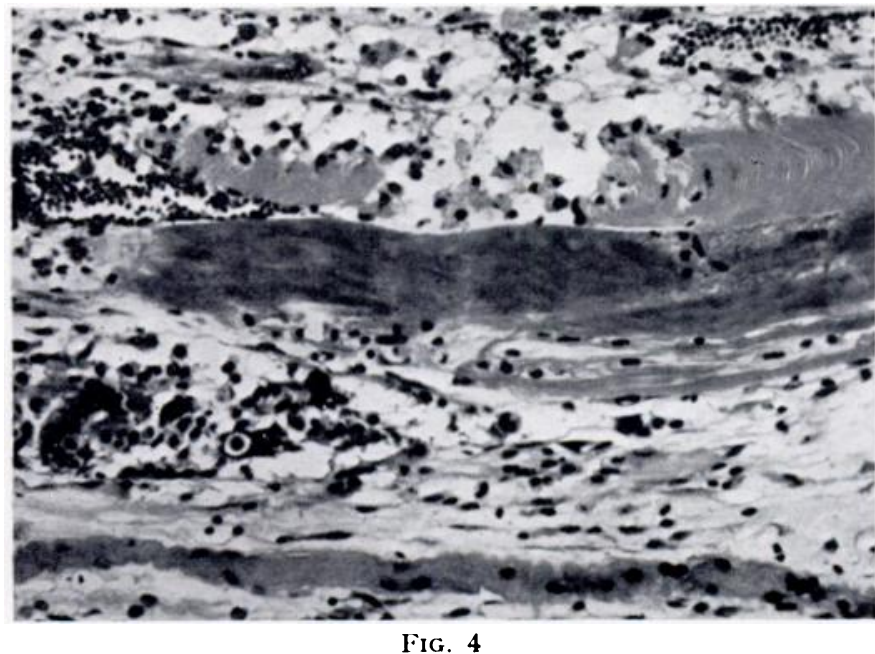

Necrotic fibres after twenty-one days; fragmentation, phagocytosis by giant cells, replacement by delicate fibrous tissue; note persistent cross striations $(\times 175)$. with narrowing of their lumina. Similar involutionary changes are known to occur in the uterine vessels after parturition, and in the umbilical cord after birth. Furthermore, with so much tissue necrosis, much of the cellular infiltration will be approximated to the vessels. It is of course recognised that sections of muscle tissue seldom contain larger vessels; but even the illustration of the anterior tibial vessels in Horn's article does not offer convincing evidence of primary arterial disease. The biopsy was taken sixteen days after the onset of necrosis; the lumen of the artery was narrowed; the media was obviously thickened; and both artery and vein were matted together in fibrous tissue. The artery at operation was found to be surrounded at one level by a mass of firm grey tissue. These changes might well have been secondary

Vol. $30 \mathrm{~B}$, No. 4 , NOVEMBER 1948 
to involvement of the vessels in the dense mass of fibrous tissue which had formed by that time. Summarising, I do not think there is anything in the histological picture which can be regarded as the cause of the muscle damage.

Pathogenesis-Study of the morbid anatomy in these cases indicates that necrosis and contracture of the muscles is the result of acute arterial insufficiency. It is well known that, in advanced arteriosclerosis of a limb in which there is peripheral gangrene, there may be complete occlusion of the femoral artery in the adductor canal and yet no more than moderate atrophy of the muscles below the knee. Occlusion of the main artery of supply will not, alone, cause muscle necrosis; the occlusion must occur rapidly and it must be maintained

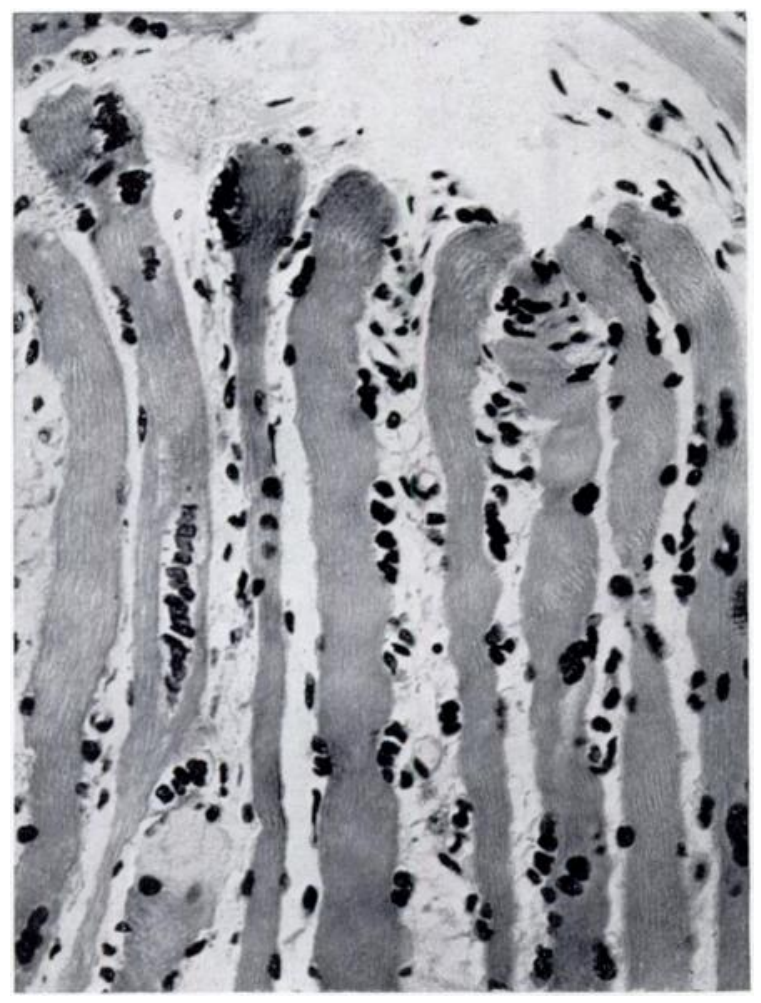

FIG. 5

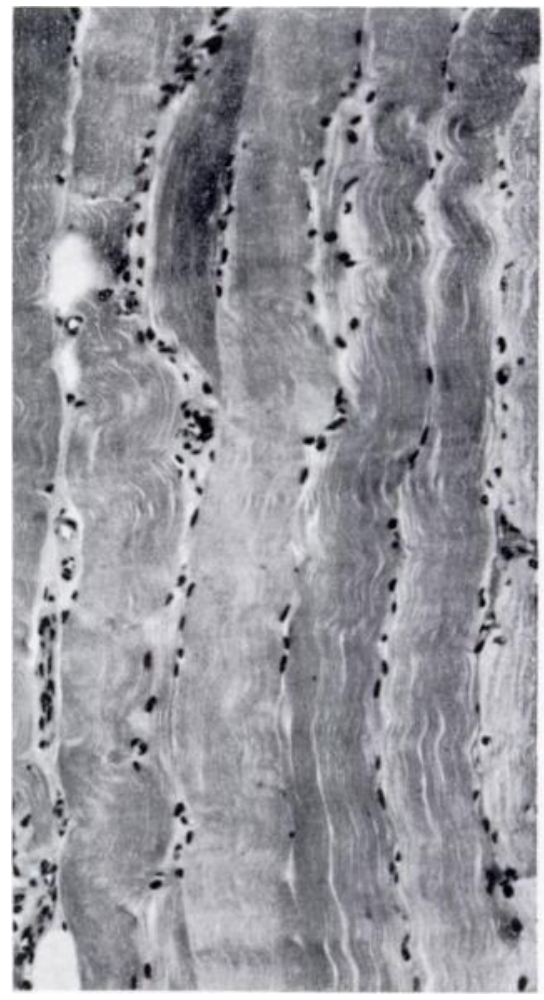

FIG. 6

Fig. 5 shows regenerating fibres of the anterior tibial muscle after necrosis in Case 1. Note the size, the nuclei, and the terminal blobs $(\times 280)$. Fig. 6 is a section from the normal gastrocnemius in the same case $(\times 150)$.

for six to twenty-four hours (Lewis 1936, Cohen 1944). Such a state could, theoretically, be brought about by extraluminary occlusion (mechanical compression or kinking) or by intraluminary occlusion (embolism, thrombosis, or spasm). Until the nature and anatomical level of such occlusion can be deduced, pathogenesis must be a matter of speculation.

Anatomical considerations in relation to vulnerability of the anterior tibial group of musclesWe may wonder why it is that the anterior tibial muscles should be so susceptible to infarction. It is not entirely a question of special demands during activity, for Case 3 illustrated bilateral involvement while the patient was confined to bed. Nor is it simple coincidence that the tibialis anterior muscle should bear the brunt of the attack, while the extensor digitorum longus muscle often escapes so lightly; it is the logical sequence of their vascular pattern. 
Blood supply of the anterior tibial muscles-Campbell and Pennefather (1919), in an excellent paper, showed that intramuscular anastomoses were so fine that the main muscular branches could be regarded functionally as end-arteries. More recently the vascular pattern of individual muscles has been worked out carefully by Blomfield (1948) using radio-opaque injection media. I am greatly indebted to him for detailed observations on the blood supply

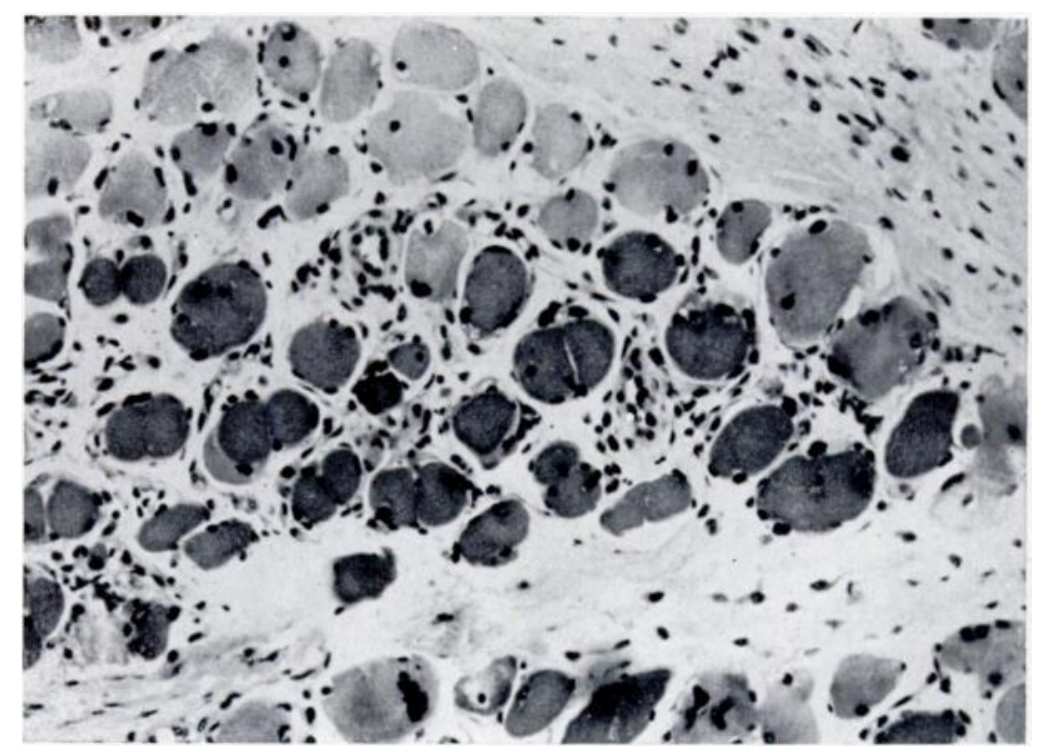

FIG. 7

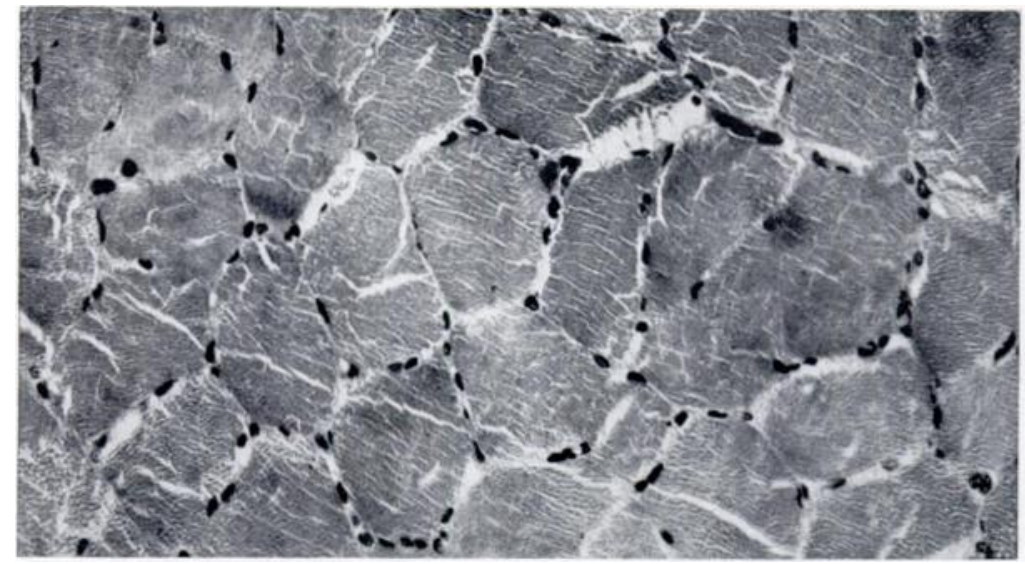

FIG. 8

Fig. 7 is transverse section of the regenerating muscle fibres in Case 1. Note the endomysial proliferaticn and variation in size. Fig. 8 is a transverse section of the normal gastrocnemius $(\times 260)$.

of the anterior tibial muscles, much of which is still unpublished. The major blood supply of muscles of the anterior tibial compartment* is from the anterior tibial artery, which emerges between the two heads of the tibialis posterior muscle. Vogt (1943) suggested that the vessels might be injured by sliding contact with the edge of the interosseous membrane.

* The term anterior tibial compartment refers to that fascial compartment containing the tibialis anterior and the two long extensors of the toes.

VOL. $30 \mathrm{~B}$, NO. 4 , NOVEMBER 1948 
This has not been substantiated in my anatomical dissections which indicated that as the vessels pass forwards they are surrounded by a cuff of loose areolar tissue and are cushioned by muscular fibres, the free edge of the interosseous membrane being situated at least one to two centimetres distally.

The tibialis anterior muscle is supplied almost entirely by the anterior tibial artery through a series of segmental branches, approximately twelve in number, which divide into ascending and descending divisions anastomosing by a system of arcades (Le Gros

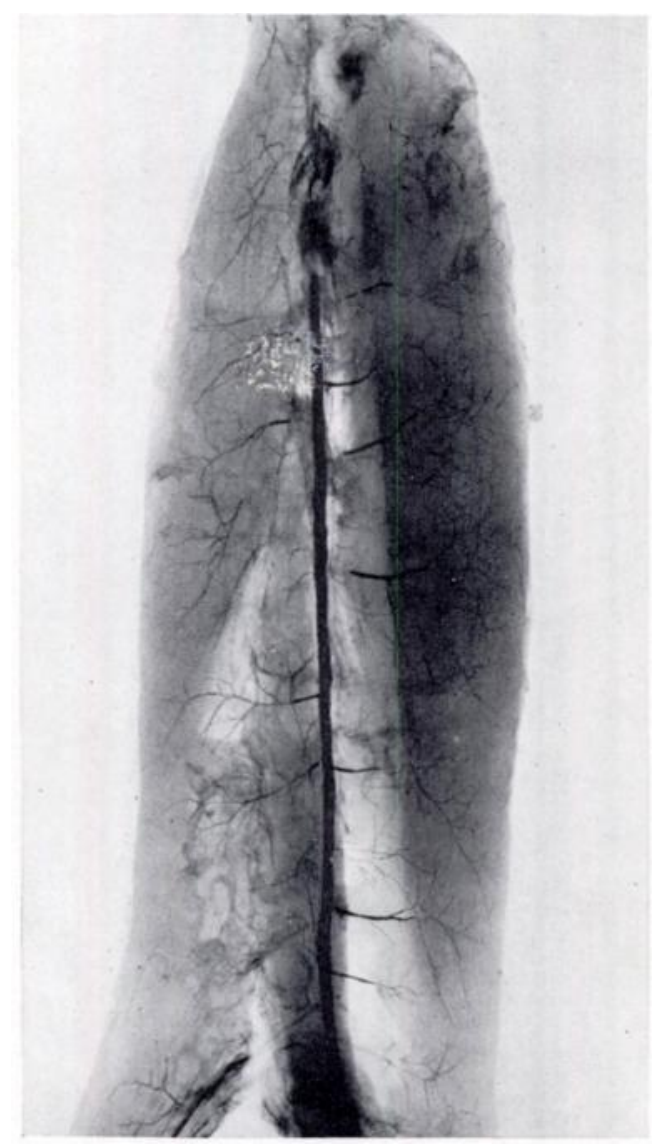

FIG. 9

(adaveric injection showing the normal vascular pattern of the anterior tibial muscles in a patient aged twenty-three years. The tibialis anterior is to the right. Radio-opaque suspension wasinjected into the femoral artery, and the anterior tibial muscles then removed en bloc.

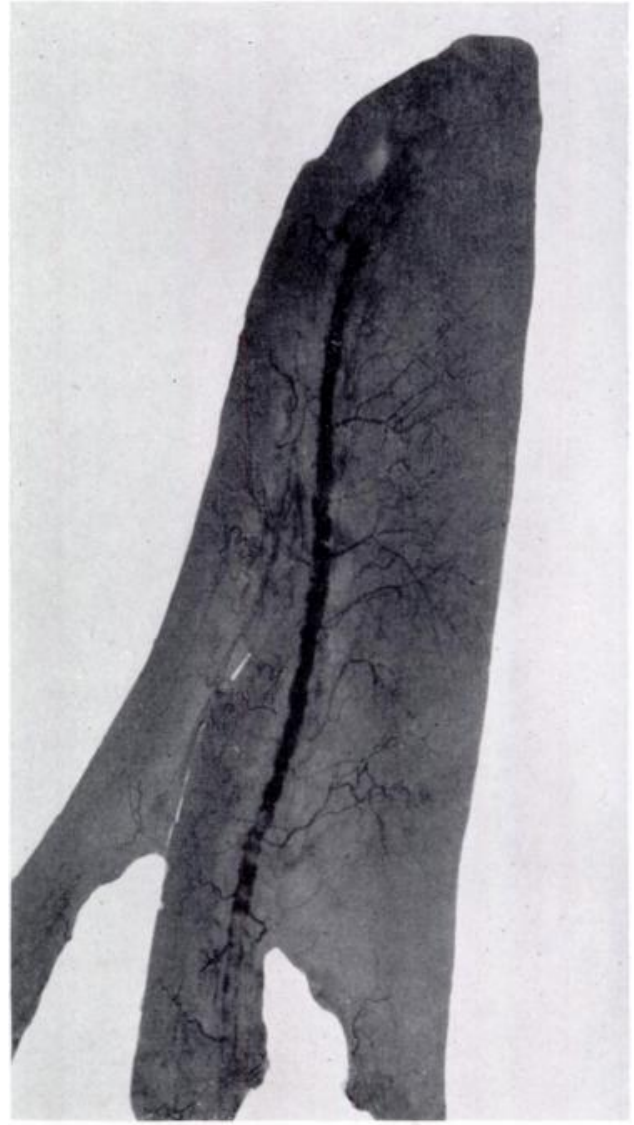

FIG. 10

Vascular pattern of the left anterior tibial muscles; injection into femoral artery after ligation of the anterior tibial artery at the level of its recurrent tibial branch. Note retrograde flow. (Patient aged seventy-nine with bilateral Paget's disease of the tibiae.)

Clark 1946, Blomfield 1948). The proximal third of the muscle is supplied by a leash from the anterior tibial recurrent artery. My own observations on injected specimens, cleared by Spaltholz' method, tend to confirm the view of Campbell and Pennefather (1919) that intramuscular anastomoses are so fine that their functional significance is open to doubt.

According to Blomfield $(1945,1948)$ the extensor digitorum longus has a threefold supply: 1) from the anterior tibial artery and its recurrent branch; 2) by three large perforating branches from the posterior tibial artery; and 3) by a large branch to the lower 
third of the muscle from the perforating peroneal artery. These branches anastomose throughout the length of the muscle. The extensor hallucis longus has a twofold supply: 1) from the anterior tibial artery; and 2) trom the perforating peroneal artery. The intramuscular anastomosis is described as rectangular and of a less efficient type (Le Gros Clark 1946).

Assuming these observations to be correct we should expect that sudden complete occlusion of the anterior tibial artery, as it enters the anterior compartment, would deprive

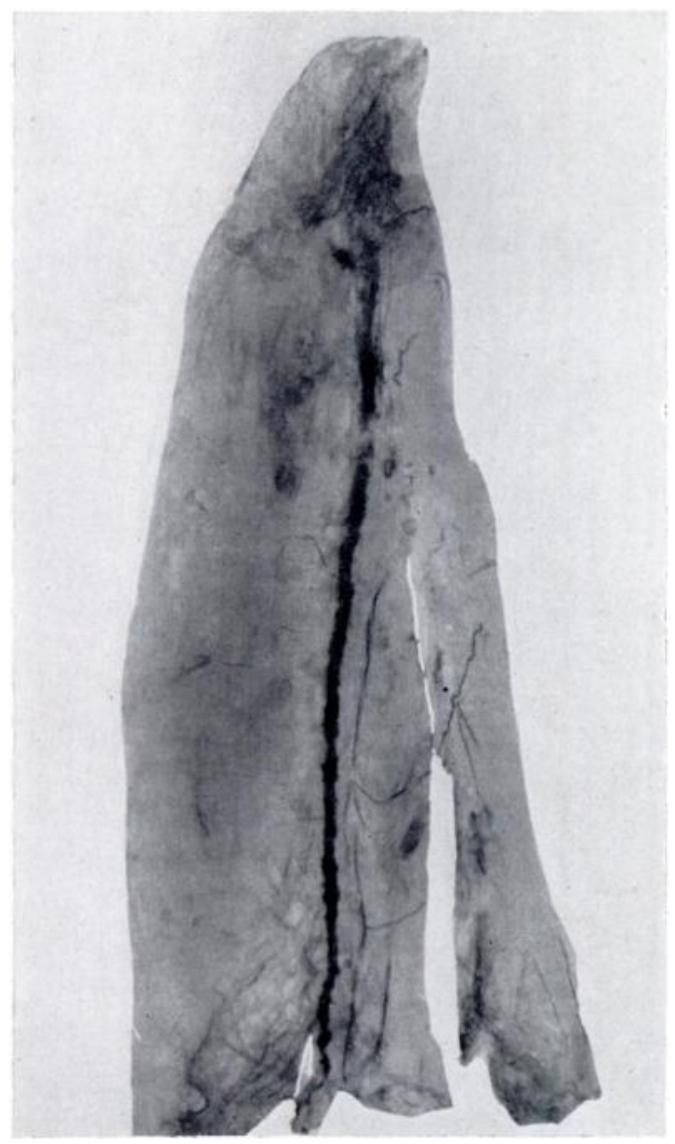

Fig. 11

Vascular pattern of right anterior tibial muscles (same case as Fig. 10); injection into femoral artery after ligation of anterior tibial artery both proximally and distally (see text). Note partial filling of anterior tibial artery through the perforating branches supplying the toe extensors.

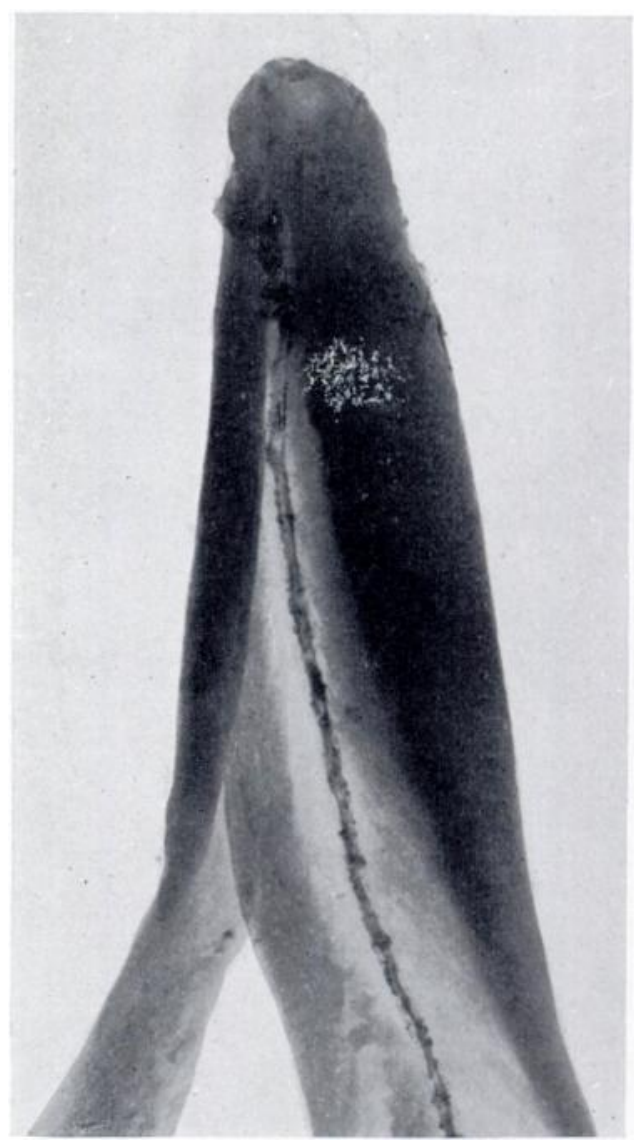

FIG. 12

Incomplete filling of the anterior tibial artery ligated proximally and distally; note very little flow into the muscles. Injection of radio-opaque suspension into the femoral artery after ligating the anterior tibial artery both proximally and distally shows incomplete filling of the main vessel and very little flow into the muscles.

the tibialis anterior of its entire blood supply and result in total necrosis. The extensor digitorum longus and extensor hallucis longus, on the other hand, having an additional blood supply from perforating arteries, might be expected to escape more lightly, the greater involvement of the long extensor of the big toe being explained by its relatively poor anastomotic network within the muscle.

It will be remembered that in two cases the clinical findings suggested that the proximal third of the muscle bellies was affected minimally. The inference to be drawn, I think, is 
that occlusion of the anterior tibial artery probably occurred distal to its tibial recurrent leash; this was certainly so in the only recorded case in which the artery was seen (Case 2).

The extreme rarity with which there is gangrene or necrosis of the peroneal muscles is accounted for by the extensive blood supply which they receive from the peroneal artery.

Tension within the fascial compartment-Much stress has been laid on the rigidity of the walls of the anterior tibial compartment and the resulting vulnerability of its contents to circulatory disturbances. Little point has been made of the fact that this fascial space is not closed at its lower end. Blomfield quoted a case reported by Zachary in which ischaemia of the anterior tibial muscles was said to have occurred after severe swelling within the fascial space. Others have been mentioned after direct blows and haematoma formation.

Lewis (1936) showed that much lower pressures than were required to collapse the main artery might result in complete arrest of the blood flow when applied directly to the muscle. Thus pressures of 50 to $60 \mathrm{~mm}$. Hg., applied directly to the muscle and maintained for twenty-four hours, might produce necrosis, whilst in animal experiments the same results were produced within six to ten hours. Prolonged activity of a muscle may increase its weight by 20 per cent., the bulk being increased by the retention of excessive fluid within the tissue spaces (Wright 1936). If for this, or any other teason, tension within the fascial compartment rises, the circulation within the intramuscular vascular networks must be embarrassed. But it must be assumed that such tension, being within a confined space, will be distributed uniformly and that all branches of the network, whether arising from the anterior tibial artery or from perforating branches, will be affected equally. On the other hand, raised pressure sufficient to obliterate the main vessel against an unyielding interosseous membrane would be more than enough to obliterate all intramuscular capillaries and give rise to uniform necrosis of all these muscles.

Sirbu, Murphy, and White (1944) described an example of ischaemic necrosis occurring five days after repair of a small hernia of the anterior tibial muscles. In such a case it is difficult to avoid the conclusion that tension played some part. The writers themselves suggested that it may have initiated arterial spasm. Nevertheless, if increased tension within the fascial compartment was the main factor in producing spontaneous ischaemic necrosis, it would be difficult to explain the susceptibility of individual muscles of the anterior tibial group, or the tendency for the proximal third of the tibialis anterior to escape.

Intraluminary vascular obstruction-The possibility of extraluminary occlusion, both in relation to the main artery and to the finer anastomotic branches, has already been discussed. It remains to consider embolism, thrombosis, and arterial spasm, as the possible causes of intraluminary obstruction. In none of the recorded cases has cardiac disease or other source of embolism been demonstrated. Furthermore, the anterior tibial artery arises from the popliteal trunk almost at a right angle, an anatomical feature which would not favour lodgement of an embolus. Learmonth (1944) described localised thrombosis of indeterminate origin affecting the main arteries of the limb, but his cases were of gradual onset and they bore little resemblance to the cases under consideration.

The evidence in favour of underlying primary arterial disease, with or without thrombosis, is not convincing. At this age, thromboangitis obliterans and periarteritis nodosa are the two most likely conditions, the former being more likely to affect vessels of the calibre of the anterior tibial artery and to occur unilaterally. Indeed Professor G. R. Cameron, in a personal communication, subscribed to this view. Case 3 in this series presented many of the clinical and histological features of periarteritis nodosa. But this disease affects the smaller vessels, and in order to produce the widespread necrosis which was observed, it would necessarily have involved most of the segmental muscular branches of the anterior tibial artery-which was not the case in any of the sections examined. In two recorded cases there was a history of previous aching in the anterior tibial region for some months, but apart from this there 
had been no symptom suggestive of underlying vascular disease. If the main vessel or its muscular branches were obliterated, one would have expected that muscle cramp or pain would have occurred during strenuous exercise, as a premonitory symptom before the onset of ischaemic necrosis.

\section{INVESTIGATIONS ON CADAVERS AS TO THE VASCULAR PATTERN OF THE ANTERIOR TIBIAL MUSCLES}

Investigations were carried out with the object of establishing the effect of arterial occlusion on the vascular pattern of the anterior tibial muscles. 1) Controls of the normal pattern were made by injecting radio-opaque suspensions, of the same viscosity as blood, into the femoral artery at a pressure of $140-200 \mathrm{~mm}$. $\mathrm{Hg}$. The anterior tibial muscles were removed en masse; their separate portions were defined and X-rayed. The muscles were then cleared, using Spaltholz' method, and the findings were confirmed (Fig. 9). 2) Adopting the same technique, injections were made into the femoral artery after ligating and dividing the anterior tibial artery at the level of the anterior tibial recurrent branch. 3) The same procedure was carried out after ligating and dividing not only the anterior tibial artery at its anterior tibial recurrent branch, but also the artery two inches proximal to the ankle joint. 4) Injections were made after ligation and division of both anterior and posterior tibial arteries, thus completely cutting off the blood supply of all three muscles. 5) The anterior tibial muscles were carefully removed in one piece together with the anterior tibial artery; the artery was then injected with a radio-opaque suspension after first ligating it one and a half inches below the point of injection. The procedure was repeated at distances of three inches, four and a half inches, and six inches distal to the point of original injection (Fig. 13).

Findings-The vascular patterns of these muscles varied with age, and they varied in different individuals. The older the subject the finer, and the more tortuous, was the intramuscular network. One subject, aged seventy-nine years, who had Paget's disease of both tibiae, exhibited a particularly generous vascular network.

Certain facts were established. 1) Occlusion of the anterior tibial artery as it enters the anterior fascial compartment does not deprive the muscles of their entire blood supply; there is often a retrograde flow from below, which is distributed to the three muscles it supplies (Fig. 10). 2) Ligation of the anterior tibial artery proximally, and also distally in order to cut off the retrograde flow, still results in seeping of blood into the intervening arterial segment which is, however, insufficient to be redistributed in significant degree to the tibialis anterior. This incomplete filling must occur through the perforating branches which pierce the extensors of the toes and sometimes effect almost direct communication with the anterior tibial trunk. The extent of the alternative supply appears to be variable. In some it is considerable and no doubt sufficient to maintain viability of the toe extensors (Fig. 11). In others, although partly filling the anterior tibial trunk, it contributes little to any of the muscles (Fig. 12). 3) Injections of the anterior tibial artery after ligation at successive levels confirms the segmental vascular arrangement and suggests that the longitudinal arcade anastomosis may not have much functional significance (Fig. 13A, B, C).

The critical level of obstruction of blood flow which causes necrosis is not known, but it would seem that a localised complete occlusion of the anterior tibial artery is unlikely to cause necrosis; on the other hand a longer segmental occlusion of the vessel, such as may be produced by arterial spasm, would obliterate the main channel by which retrograde distribution through the segmental branches may be brought about. The alternative blood supply to the two extensors through perforating branches may be sufficient in some cases to preserve their viability but quite apart from this their smaller bulk tends to minimise the degree of contracture which can be detected clinically.

vol. $30 \mathrm{~B}$, No. 4, NOVEMBER 1948 
Relationship to exercise-The relationship of exercise to acute ischaemia of the anterior tibial muscles is so striking a feature that it demands consideration. Horn (1945) ingeniously surmised that "hypertrophy and fibrosis of the media of the anterior tibial artery" which he believed to be an essential underlying vascular change, was a result of the repeated and overwhelming demands of muscles during strenuous exercise. He believed that involvement of the adventitia in this fibrosis caused reflex vasoconstriction " affecting the entire collateral network " and that the circulation was further impaired by muscular swelling due to chemical and histological changes as described by Howard (1937) in crepitating tenosynovitis. This hypothesis does not, however, bear critical scrutiny. No artery narrows its lumen in response

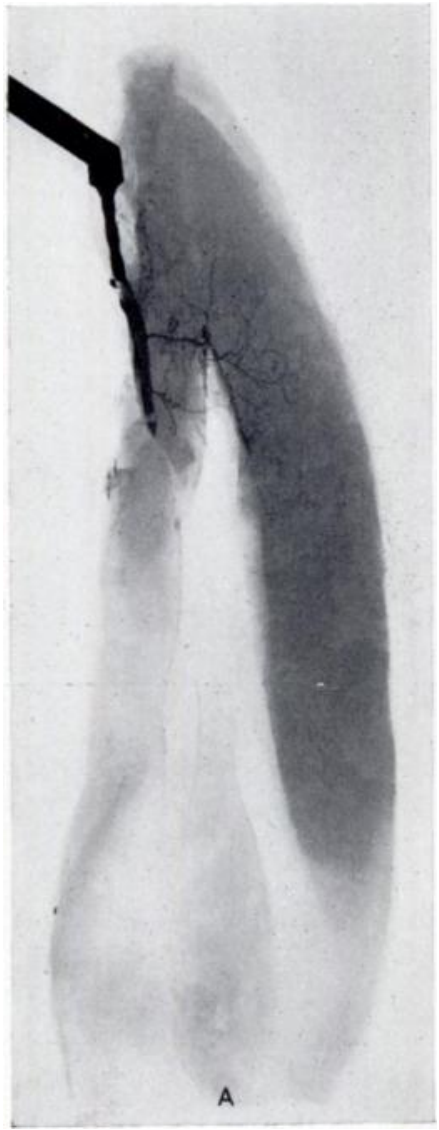

$A, B$, and $C$ show the vascular pattern after ligation of the anterior tibial artery $1 \frac{1}{2}$ inches, 3 inches and $4 \frac{1}{2}$ inches respectively, from the point of injection (see text); note the segmental nature of the blood supply.

to increased demands by the area it supplies. The normal vascular response to muscular activity is one of diminished peripheral resistance with an increased capillary bed. There is no hypertrophy of the media; such a change, if it occurred, would be more in keeping with prolonged arterial spasm. Secondly, the tibialis anterior has no collateral blood supply other than its segmental branches, and even in those muscles which have an alternative supply the intramuscular networks are probably under humoral rather than autonomic control. Thus spasm, even if segmental, does not presuppose involvement of a collateral field in order to produce complete ischaemia.

Whatever condition may be responsible for acute ischaemia it must have persisted for six

THE JOURNAL OF BONE AND JOINT SCRGERY 
to twenty-four hours in order to produce necrosis (Lewis 1936, Cohen 1944), and there is clinical evidence that the resulting inflammatory reaction occurs about twelve to twenty-four hours after the strenuous exercise, suggesting that vascular insufficiency began during the physical effort, or probably shortly after it. In Case 1 and Case 2 in this series the onset appears to have been after exercise; on the other hand Horn's cases suggest onset during the exercise.

It may be urged that complete vascular occlusion need not be assumed, and that the ischaemia is relative rather than absolute. Demands upon the vascular supply may be in excess of that which it can provide, for the metabolism of muscle in activity is greatly increased, and if exercise is more strenuous than is customary for the individual, the power of oxygen abstraction is appreciably diminished (Wright 1936). The muscle is subjected to relative anoxia and necrosis supervenes. The analogy to this would be the ischaemic limb in which gangrene is precipitated by increasing the metabolic demands of its tissues. Such a view is hardly tenable when one realises that in order to produce muscle necrosis the oxygen deprivation would have to persist much longer than the period of muscular exercise to which the patients were subjected. Nevertheless, I think that relative anoxia during strenuous and unaccustomed exercise is probably an important secondary factor in tipping the scales in favour of necrosis. The local accumulation of lactic acid, and its coagulative action on muscle fibres as described by Albert and Mitchell (1943), whether it be the result of venous obstruction or of arterial insufficiency, is merely the means by which anoxia effects necrosis.

In the light of present knowledge it seems that the most likely primary cause of spontaneous ischaemia is spasm of a large segment of the anterior tibial artery, beginning just below its anterior tibial recurrent branch. Is it possible that the marked chemical and $\mathrm{pH}$ changes which are known to occur in fatigued muscles, and in tenosynovitis, could initiate this spasm? It is known that muscle break-down products in ischaemia, particularly adenosine derivatives, are capable of producing spasm of the renal vessels (Green and Stoner 1945), and it may well be that accumulated metabolites in fatigued muscle are capable of a similar action locally. Tension within the anterior fascial compartment is probably an aggravating factor. Arteriography, or exploration within the first twelve hours after exercise, may provide the answer.

\section{TREATMENT}

Advance in our knowledge of curative treatment depends upon early diagnosis. The obvious difficulty lies in the fact that if there is delay, irreversible damage has already been done by the time that inflammatory changes become evident clinically. The object should be to relieve arterial spasm before necrosis supervenes. Early eupaverine injection might be helpful, but the effect of paravertebral block on muscle circulation is still debatable. Assuming that a case is seen within the first twelve hours, exploration appears to be warranted. The operative approach should be between the muscles rather than through them. Care must be taken to avoid damage to intramuscular branches. The fascia cruris should not be repaired. If there is arterial spasm the appropriate treatment should be employed but it must be borne in mind that arteriectomy should be limited to resection of a very small segment; the part of the tibialis anterior muscle which will be deprived permanently of its blood supply is directly proportionate to the length of arterial segment excised. The danger of exploring muscles after necrosis has supervened cannot be over-emphasized. Necrotic tissues are prone to infection and gangrene, and in one case amputation was necessary. (Sirbu et al. 1944.)

Whatever treatment may be undertaken during the first twenty-four hours, the limb should be supported with the ankle in the mid-position and free from constriction. This position should be maintained until the acute inflammatory reaction has subsided and until there is no longer any tendency to further contracture. Subsequently a toe-elevating spring should be worn for the first few months of weight-bearing. Surviving muscle fibres should be redeveloped by graduated exercise.

Vol. $30 \mathrm{~B}$, No. 4 , NOVEMBer 1948 


\section{SUMMARY}

1. Three cases are reported of ischaemic necrosis of the anterior tibial muscles which were not due to injury. In two, ischaemia was the result of strenuous or unaccustomed exercise in young adults; in the third it was an incident in a systemic disturbance. All three cases were probably the result of spasm of a large segment of the anterior tibial artery. 2. The clinical features during the first few hours resemble those of tenosynovitis of the tibialis anterior; and after twelve to twenty-four hours those of cellulitis of the leg. Later there is " drop foot " due to muscle weakness, contracture limiting plantar-flexion movement, and woody hardness of the muscles in the middle third.

3. The morbid histology is similar to that of Volkmann's ischaemic contracture.

4. The possible explanations-primary arterial disease, arterial occlusion by pressure of the interosseous membrane, occlusion by tension within the fascial space, intraluminary occlusion by embolism or thrombosis, and fatigue arterial spasm, are discussed.

5. The vascular pattern of the anterior tibial muscles has been studied by experimental injections in cadavers.

6. It is concluded that the most likely cause is spasm of the anterior tibial artery due to muscle fatigue, aggravated by increased tension within the anterior fascial compartment due to reaction after strenuous exercise.

7. Treatment is outlined. Exploration of the anterior tibial artery within the first twelve hours is warranted, but late exploration may be dangerous.

8. Although not previously recognised, evidence is shown that regeneration of necrotic muscle is possible in the human being.

I wish to express my thanks to Sir Reginald Watson-Jones for his encouragement, and to him and $\mathrm{Mr}$ $H$. Osmond-Clarke for permission to publish these cases; to Dr Harrison, Professor G. R. Cameron, and Professor Le Gros Clark for their advice on histological material; in particular to Dr Blomfield for access to unpublished work on the vascular pattern of muscles; to Mr R. Tucker, Lady Jones Research Fellow in Liverpool, for injection facilities and for valuable help and criticism; and to Mr John King of the London Hospital to whom I am indebted for the microphotographs.

\section{REFERENCES}

Albert, M., and Mitchell, W. R. D. (1943): Lancet, 1, 519.

Blomfield, L. B. (1945): Proceedings of the Royal Society of Medicine (Section of Orthopaedics), $38,617$. Blomfield, L. B. (1948): Personal communication.

Bowden, R. E. M., and Gutmann, E. (1945): Lancet, ii, 768.

BRooks, B. (1922): Archives of Surgery, 5, 188.

BRUCE, J. (1940): Journal of Bone and Joint Surgery, 22, 738.

Cameron, G. R. (1948): Personal communication.

Campbell, J., and Pennefather, C. M. (1919): Lancet, 1, 294.

Clark, W. E. Le Gros (1946): Journal of Anatomy, 80, 24.

Clark, W. E. Le Gros (1948): Personal communication.

Clark, W. E. Le Gros (1946): Bulletin of War Medicine, 6, 267.

Clark, W. E. Le Gros, and Blomfield, L. B. (1945): Journal of Anatomy, 79, 15.

Cohen, S. M. (1944): Lancet, $1,1$.

Green, H. N., and Stoner, H. B. (1945): Journal of Pathology and Bacteriology, 57, 337.

Griffiths, D. Ll. (1940): British Journal of Surgery, 28, 239.

Hill, R. L., and Brooks, B. (1936): Annals of Surgery, 103, 444.

HoRN, C. E. (1945): Journal of Bone and Joint Surgery, 27, 615.

HowARD, N. J. (1937): Journal of Bone and Joint Surgery, 19, 447.

Learmonth, J. R., Blackwood, W., and Richards, R. L. (1944): Edinburgh Medical Journal, $51,1$.

Lewis, Sir Thomas (1936): Vascular Disorders of the Limbs. London: Macmillan \& Co., 36.

Middleton, D. S. (1930): British Journal of Surgery, 18, 188.

Parkes, A. R. (1945): British Journal of Surgery, 32, 403.

Power, R. W. (1945): British Medical Journal, 1, 656.

Sirbu, A. B., Murphy, M. J., and White, A. S. (1944): California and Western Medicine, 60, 53.

VoGT, P. R. (1943); Ischaemic Muscular Necrosis following Marching. Unpublished, but read before the Oregon State Medical Society, September 4, 1943; and quoted by HoRn, C. E. (1945) vide supra. Wright, Samson (1936): Applied Physiology, 6th edition. London: Oxford University Press. 